\title{
Cardiothoracic
}

Transplantation

\section{Optimal timing of cardiac transplantation after ventricular assist device implantation}

\author{
James S. Gammie, $\mathrm{MD}^{\mathrm{a}}$ \\ Leah B. Edwards, $\mathrm{PhD}^{\mathrm{b}}$ \\ Bartley P. Griffith, $\mathrm{MD}^{\mathrm{a}}$ \\ Richard N. Pierson III, MD \\ Lana Tsao, MD ${ }^{\mathrm{c}}$
}

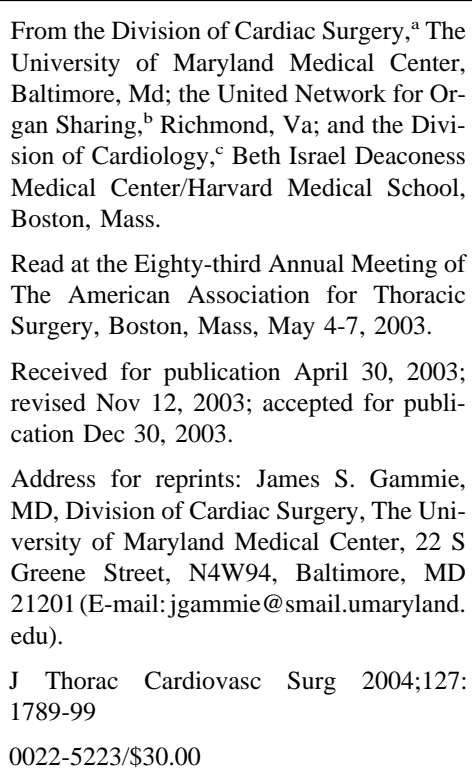

Copyright () 2004 by The American Association for Thoracic Surgery

doi:10.1016/j.jtcvs.2003.12.018
Objective: We sought to determine the influence of the interval from ventricular assist device implantation to cardiac transplantation on end-organ function and posttransplantation survival.

Methods: United Network for Organ Sharing data on 2692 heart transplantations performed in adult patients in the United States between October 1999 and March 2001 were reviewed.

Results: Seventeen percent (466) of adult heart transplant recipients were bridged to transplantation with a ventricular assist device. Almost half of patients with ventricular assist devices undergoing transplantation were upgraded to status $1 \mathrm{~A}$ as a result of ventricular assist device-related complications occurring more than 30 days after ventricular assist device implantation. Creatinine and total bilirubin levels were less in patients undergoing transplantation after 2 to 4 weeks of mechanical support. One-year survival was higher in the nonventricular assist device than in the ventricular assist device group $(85.7 \%$ vs $79.7 \%, P=.0004)$. Within the ventricular assist device group, survival was lower for patients undergoing transplantation within 2 weeks of ventricular assist device implantation compared with those undergoing transplantation later $(74.2 \%$ vs $84.2 \%, P=.03)$. One-year survival among patients supported with a ventricular assist device for more than 30 days without complications was $91.4 \%$. Multivariate analysis demonstrated a significant independent effect of the time interval from ventricular assist device implantation to transplantation on posttransplantation mortality and suggested that a period of lowest risk might exist between 1 and 3 months after implantation.

Conclusions: Survival after cardiac transplantation is influenced by the time interval from ventricular assist device insertion to transplantation. Survival is significantly lower when performed within 2 to 4 weeks of ventricular assist device implantation.

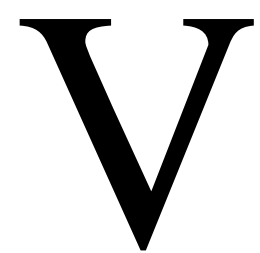

entricular assist devices (VADs) are commonly used to bridge the patient with end-stage heart failure to cardiac transplantation. Given the critically ill population for whom they are used, outcomes reported in single-center series are remarkably favorable: $70 \%$ to $80 \%$ of patients undergoing VAD implantation survive to receive a heart transplantation, and survival after transplantation is similar to that of unsupported recipients. ${ }^{1}$ The decision regarding timing of 
cardiac transplantation for an individual device-dependent patient is challenging and is based on multiple considerations, including the availability of donor hearts, the presence of device-related complications, and the physiologic state of the recipient. Although mortality is high in the first few weeks after VAD implantation, ${ }^{2}$ the benefits of early removal of a potentially morbid device and transplantation might be balanced by the value of longer-term rehabilitation that a device affords the patient. Few published data are available to guide decision making. Previous reports have shown conflicting results of the effect of timing of transplantation after VAD insertion on rates of survival after transplantation. ${ }^{3,4}$ In October 1999, the United Network for Organ Sharing (UNOS) allocation algorithm for hearts was changed from a 2-tier to a 3-tier system. Under the new algorithm, VAD-supported patients were assigned to the highest priority class for 30 days after VAD insertion, after which after they were downgraded to a middle-tier urgency status in the absence of device-related complications. ${ }^{5} \mathrm{We}$ sought to define the prevalence of VAD use in the United States, quantitate the demographics of the supported population reaching transplantation, and determine whether the interval from VAD implantation to transplantation exerts an independent influence on posttransplantation survival under the modified allocation system.

\section{Methods}

Posttransplantation analyses were based on all heart transplantations performed in adult patients in the United States between October 25, 1999, and March 30, 2001 ( $\mathrm{n}=2692)$, as reported to UNOS, excluding multiorgan and heterotopic heart recipients. Analyses were based on UNOS data as of September 2002. The mean follow-up after transplantation was 515 days (range, 0-988 days, \pm 215 days).

In the new (October 1999) UNOS allocation system, patients are considered status $1 \mathrm{~A}$ for the first 30 days after device insertion, at which time they are downgraded to status 1B. Development of device-related complications, such as infection or thromboembolism, upgrades the candidate to status $1 \mathrm{~A} .{ }^{5}$ The medical urgency status categories used in the analyses are defined as follows:

1. 1A(a)-VAD: left ventricular assist device (LVAD), right ventricular assist device, or both implanted for 30 days or less;

2. 1A(a)-other: total artificial heart, intra-aortic balloon pump, or extracorporeal life support;

3. 1A(b)-VAD with complications: mechanical circulatory support for more than 30 days with evidence of significant device-related complications, including thromboembolism, device infection, mechanical failure, and/or life-threatening ventricular arrhythmias;

4. 1A-nonVAD: mechanical ventilation, high-dose intravenous inotropes, or life expectancy of less than 7 days;

5. 1B(a)-VAD $>30$ days: LVAD, right ventricular assist device, or both implanted for more than 30 days;

6. 1B-nonVAD: low-dose inotropes; and
7. Status 2: a patient who does not meet the criteria for status $1 \mathrm{~A}$ or $\mathrm{B}$.

The date of VAD implantation is currently collected by using justification forms for status $1 \mathrm{~A}$ and $1 \mathrm{~B}$ criteria. These forms are completed when a patient is initially placed on the waiting list in status $1 \mathrm{~A}$ or $1 \mathrm{~B}$, upgraded to one of these statuses, remains on the waiting list in status $1 \mathrm{~A}$ or $1 \mathrm{~B}$ for an extended period of time and requires an extension, or remains on the waiting list in one of these statuses but the criteria for their status have changed. Before October 25, 1999, status justification data were submitted by centers to UNOS by paper, and the data were maintained primarily for monitoring policy compliance. As such, the VAD implantation date was reviewed by compliance staff but was not retained in the patient's electronic record at UNOS. Between October 25, 1999, and August 2000, centers had the option of submitting the status justification data by paper or through UNOS's electronic data entry system. After August 2000, electronic submission of status justification data was required. Twenty-eight percent (132/466) of VAD-supported patients did not have a recorded date of implantation. For this group, the interval from VAD implantation to transplantation was not defined, although their medical urgency status category identified whether the duration of support was more or less than 30 days.

A waiting-list analysis was performed on all adult heart transplantation candidates added to the waiting list between October 25, 1999 and December 31, 2001, to quantitate the risk of death or removal from the waitlist during VAD support. Only patients spending at least 1 day in status $1 \mathrm{~A}$ or $1 \mathrm{~B}$ were included. The resulting cohort included 5091 new registrations.

Comparisons of percentages between groups were made by using the $\chi^{2}$ test. Because of concerns regarding nonnormality, comparisons of the mean for continuous variables, such as age or creatinine level, were made by using the Wilcoxon test. Posttransplantation survival rates were computed by using the KaplanMeier method; rates were compared by using the log-rank test statistic.

A multivariate proportional hazards model was developed on the basis of the transplant recipients to determine factors having an effect on posttransplantation survival. The effect of the time between VAD implantation and transplantation was analyzed by using a restricted cubic spline.

Waiting list outcomes were computed by using a competing risks extension of the Kaplan-Meier method. ${ }^{6}$ The following events were analyzed: death on the waiting list, transplantation, removal from the waiting list for reasons other than death, or transplantation. For patients with a VAD at any time during their waiting period, the percentage of each event was computed from the later of the time of VAD implantation or placement on the waiting list.

\section{Results \\ Demographics}

Medical urgency status at the time of cardiac transplantation is outlined in Table 1. During the 17-month time period studied, 74\% (1986/2692) of all recipients were status 1A or status $1 \mathrm{~B}$ at the time of transplantation, and a VAD was in place in $23 \%$ (466/1986). Overall, 17\% (466/2692) of adult 
TABLE 1. Medical urgency status at the time of cardiac transplantation

\begin{tabular}{lcc}
\hline Status & $\mathbf{n}$ & $\%$ \\
\hline 1A(a)-VAD & 169 & 6.3 \\
1A(a)-other (IABP, ECLS) & 139 & 5.2 \\
1A(b)-VAD with complication & 222 & 8.2 \\
1A-nonVAD (high inotropes, & 444 & 16.5 \\
$\quad$ vent, life $<7 \mathrm{~d}$ ) & & \\
1B(a)-VAD $>30 \mathrm{~d}$ & 75 & 2.8 \\
1B-nonVAD (low inotropes) & 937 & 34.8 \\
2 & 706 & 26.2 \\
Total & 2692 & 100 \\
\hline
\end{tabular}

$\overline{V A D}$, Ventricular assist device; IABP, intra-aortic balloon pump; $E C L S$, extracorporeal life support.

heart transplant recipients were bridged to transplantation with a VAD. Among patients transplanted with a VAD in place, 36\% (169/466) underwent transplantation within 30 days of VAD implantation (1A[a]-VAD), 48\% (222/466) underwent transplantation more than 30 days after VAD implantation with a device-related complication (1A[b]VAD with complications), and $16 \%$ (75/466) were transplanted more than 30 days after VAD implantation free of a device-related complication (1B[a]-VAD $>30$ days). The median time from VAD implantation to transplantation by medical urgency status at transplantation was 18 days for group $1 \mathrm{~A}(\mathrm{a})-\mathrm{VAD}, 101$ days for group $1 \mathrm{~A}(\mathrm{~b})-\mathrm{VAD}$ with complications, and 92 days for group $1 \mathrm{~B}(\mathrm{a})-\mathrm{VAD}>30$ days.

\section{Characteristics of VAD and non-VAD Patients}

Use of VADs was similar in male (361/2046 [17.6\%]) and female (105/646 [16.3\%]) patients. Patients with VADs were slightly younger (mean of 50.5 and 52.9 years, respectively; $P<.0001$ ). VAD-supported patients were more likely to have type $\mathrm{O}$ blood group $(48.3 \%)$ than those in the non-VAD group $(33.5 \%, P<.0001)$. Blood group A was less common in the VAD group (34.8\%) than in the nonVAD group $(46.2 \%, P<.0001)$.

Use of VADs was similar in patients with a diagnosis of ischemic cardiomyopathy (237/1348 [17.6\%]) as among those with nonischemic cardiomyopathy (201/1138 [17.7\%]). There was a statistically significant difference in both the average height (174.7 vs $173.5 \mathrm{~cm}, P=.03$ ) and weight (81.3 vs $79.3 \mathrm{~kg}, P=.01)$ of VAD-supported patients when compared with patients without VADs. The VAD-supported patients had a higher panel-reactive antibody (PRA) at the time of transplantation when compared with patients without a VAD $(P<.0001)$, although the average was low for both groups $(7.2 \%$ vs $3.0 \%$, respectively).

Donor characteristics (including age, ischemic time, cause of death, and other factors) were not significantly
TABLE 2. Comparison of donor and transplant characteristics between transplantations in VAD-supported patients versus non-VAD supported patients

\begin{tabular}{|c|c|c|c|}
\hline & $\begin{array}{l}\text { VAD patients } \\
\text { (n= } \mathrm{n}=466)\end{array}$ & $\begin{array}{c}\text { Non-VAD patients } \\
(\mathrm{n}=2226)\end{array}$ & $P$ valuet \\
\hline \multicolumn{4}{|l|}{ Characteristic ${ }^{*}$} \\
\hline Donor age (y) & 32.48 & 32.55 & .9 \\
\hline Ischemia time $(\mathrm{h})$ & 3.11 & 3.07 & .2 \\
\hline $\begin{array}{l}\text { Donor total bilirubin (mg/ } \\
\mathrm{dL} \text { ) }\end{array}$ & 0.98 & 0.99 & .9 \\
\hline $\begin{array}{l}\text { Donor serum creatinine } \\
\quad(\mathrm{mg} / \mathrm{dL})\end{array}$ & 1.05 & 1.00 & .01 \\
\hline \multicolumn{4}{|l|}{ Donor characteristic $\ddagger$} \\
\hline \multicolumn{4}{|l|}{ Cause of death } \\
\hline Anoxia & $6.7 \%$ & $6.6 \%$ & .2 \\
\hline Cerebrovascular & $31.6 \%$ & $32.1 \%$ & \\
\hline Head trauma & $60.0 \%$ & $57.7 \%$ & \\
\hline CNS tumor & $1.3 \%$ & $1.2 \%$ & \\
\hline Other & $0.4 \%$ & $2.1 \%$ & \\
\hline $\begin{array}{l}\text { Clinical infection at time of } \\
\text { recovery }\end{array}$ & $25.5 \%$ & $21.4 \%$ & .06 \\
\hline $\begin{array}{l}\text { Cigarette use }>20 \text { pack- } \\
\text { years }\end{array}$ & $37.9 \%$ & $37.3 \%$ & .8 \\
\hline History of cocaine use§ & $14.8 \%$ & $10.9 \%$ & .02 \\
\hline Pretreatment steroids & $55.7 \%$ & $57.5 \%$ & .5 \\
\hline History of diabetes & $2.4 \%$ & $1.8 \%$ & .4 \\
\hline History of hypertension & $12.8 \%$ & $11.7 \%$ & .5 \\
\hline
\end{tabular}

Percentages were computed on the basis of known values only. Factors with $P$ values of less than .1 are italicized.

$V A D$, Ventricular assist device; $C N S$, central nervous system.

* Mean per group.

† $P$ values were computed by using the Wilcoxon test for continuous factors and the $\chi^{2}$ test for categoric factors.

$\ddagger$ Percentages within group.

§Cocaine use collected on donors only since October 25, 1999.

different between VAD-supported patients and patients without VAD (Table 2).

\section{VAD Type}

The types of VADs implanted are outlined in Figure 1. Implanted (intracorporeal) LVADs were the most common configuration for hemodynamic support during bridge to transplantation, composing nearly $75 \%$ of VADs reported during this time period.

\section{Time to Transplantation}

Survival. One-year survival of the 2692 patients undergoing cardiac transplantation during the study was $84.7 \%$. As a group, patients bridged to transplantation with a VAD had a lower 1-year survival compared with nonbridged patients $(79.7 \%$ vs $85.7 \%, P=.0004)$. Subgroups of VADsupported recipients enjoyed survival that was similar to that of non-VAD recipients: 1-year survival after transplantation was $91.4 \%$ for status $1 \mathrm{~B}(\mathrm{a})$ recipients (VAD $>30$ days without complications; Table 3 ). 


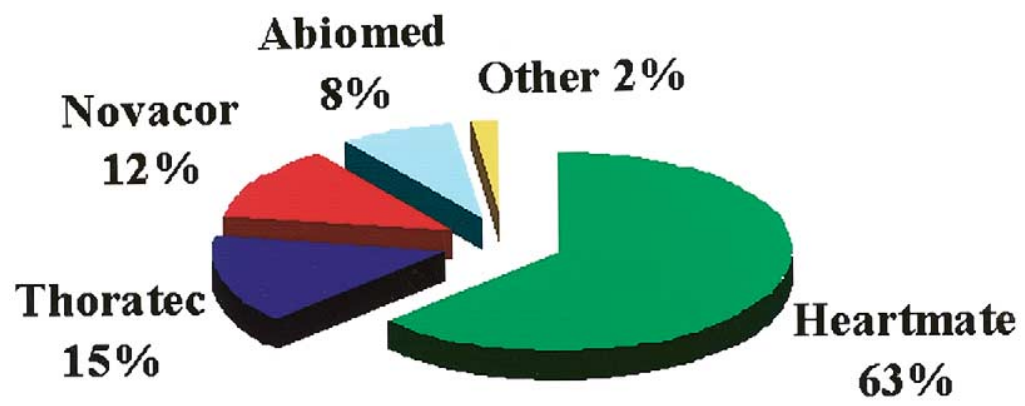

Figure 1. Type of VAD.

TABLE 3. One-year survival on the basis of medical urgency at the time of transplantation

\begin{tabular}{lc}
\hline Status & Survival (\%) \\
\hline 1A(a)-VAD & 79.1 \\
1A(a)-other (IABP, ECMO) & 75.6 \\
1A(b)-VAD with complication & 76.3 \\
1A-nonVAD (high inotropes, vent, life $<7$ d) & 85.3 \\
1B(a)-VAD >30 d & 91.4 \\
1B-nonVAD (low inotropes) & 86.9 \\
2 & 86.5 \\
\hline
\end{tabular}

$V A D$, Ventricular assist device; IABP, intra-aortic balloon pump; ECMO, extracorporeal membrane oxygenation.

Although the 1-year survival rate for this group was higher than that for all other patients combined (84.5\%), this difference was not statistically significant $(P=.2)$.

There was a clear relationship between the duration of VAD support and posttransplantation survival (Table 4). One-year survival was significantly lower for patients undergoing transplantation within 2 weeks of VAD implantation compared with those undergoing transplantation later $(P=.03$ for $0-2$ weeks vs $>2$ weeks). There was at least a $10 \%$ decrease in perioperative survival when transplantation was performed less than 2 weeks or more than 6 months from VAD implantation.

The results of a multivariate proportional hazards model developed to determine factors having an effect on posttransplantation survival are outlined in Table 5 and Figures 2 to 4 . The presence of VAD complications (ie, status $1 \mathrm{~A}[\mathrm{~b}])$ and donor-recipient sex mismatch were found to be independent predictors of increased posttransplantation mortality. There is a significant independent effect of the time interval from implantation to transplantation on survival, with a rapid decrease in the relative risk in the first 30 days after VAD implantation and a period of lower relative risk between 30 and 70 days after implantation (Figure 2). Bilirubin and creatinine levels at transplantation both exerted an independent effect on 1-year posttransplantation survival (Figure 3).
TABLE 4. One-year survival on the basis of duration of VAD support

\begin{tabular}{lccc}
\hline $\begin{array}{l}\text { Time from implantation to } \\
\text { transplantation }\end{array}$ & N & 1-mo survival (\%) & 1-y survival (\%) \\
\hline 0-2 wk & 59 & 86.4 & 74.2 \\
2-4 wk & 58 & 96.6 & 83.8 \\
4-6 wk & 70 & 97.1 & 92.4 \\
6-12 wk & 34 & 88.0 & 85.0 \\
12 wk-6 mo & 71 & 94.2 & 83.6 \\
$>6$ mo & 42 & 83.1 & 75.8 \\
\hline
\end{tabular}

TABLE 5. Multivariate analysis of 1-year posttransplantation survival in the bridge-to-transplant population

\begin{tabular}{lccc}
\hline Risk factor & Relative risk & $\boldsymbol{P}$ value & $\begin{array}{c}\mathbf{9 5 \%} \text { confidence } \\
\text { limits }\end{array}$ \\
\hline VAD complications & 1.75 & .08 & $0.93,3.30$ \\
$\begin{array}{l}\text { Sex mismatch } \\
\text { Time from implantation to } \\
\quad \text { transplantation }\end{array}$ & 1.59 & .03 & $1.05,2.39$ \\
$\begin{array}{l}\text { Bilirubin } \\
\text { Creatinine }\end{array}$ & .07 & \\
& See Figures & .03 & \\
& $1-3$ & & \\
& & .06 &
\end{tabular}

VAD, Ventricular assist device.

*Factors that were analyzed but that were not significant in the model include the following-recipient factors: race, sex, peak panel-reactive antibody, and most recent panel-reactive antibody; donor factors sex, age, donor body mass index, cigarette use, history of hypertension, clinical infection at recovery, steroid use during recovery, cause of death; transplant factors; donor/recipient body mass index ratio, center volume, and donor-recipient cytomegalovirus mismatch.

End-organ function. Table 6 outlines end-organ function and PRA levels on the basis of duration of mechanical circulatory support. There was clear improvement in both renal and hepatic function as the duration of support increased. As expected, PRA levels at the time of transplantation were higher in those patients supported longer.

When categorized by medical urgency status, we found that creatinine level before transplantation was significantly less among patients supported with a VAD for more than 30 


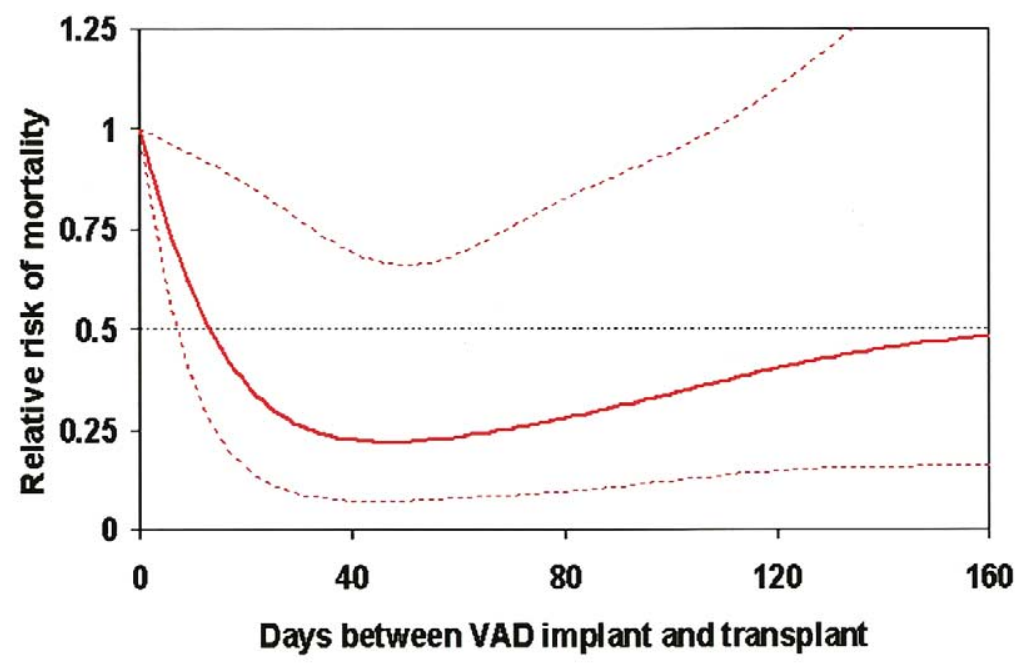

Relative risk with $95 \%$ confidence intervals

Figure 2. Effect of time between VAD implantation and transplantation on posttransplantation mortality.

Relative risk with $95 \%$ confidence intervals
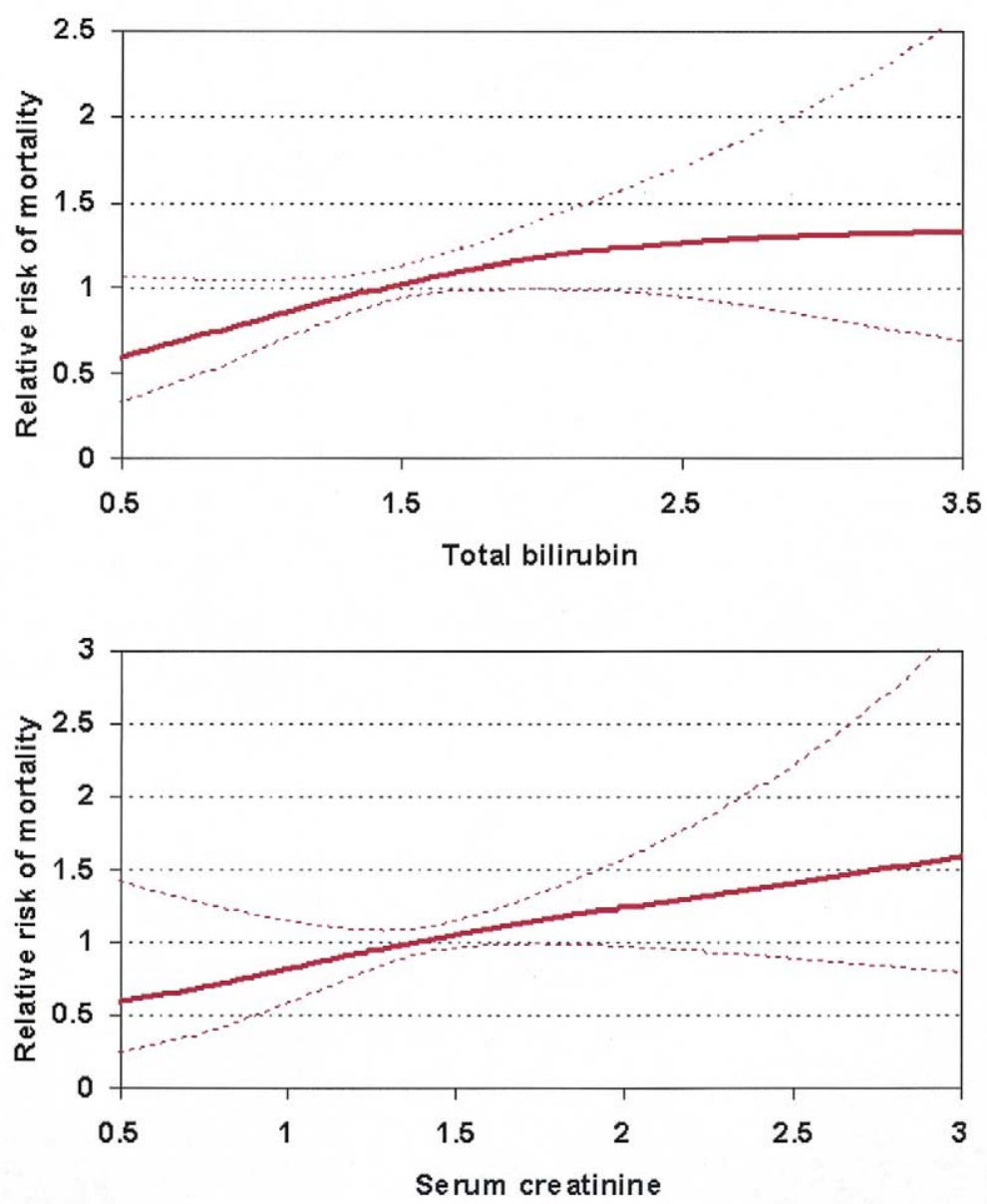

Figure 3. Effect of recipient serum creatinine and total bilirubin levels on posttransplantation mortality. 
TABLE 6. End-organ function and sensitization as a function of time on device support

\begin{tabular}{lcccc}
\hline $\begin{array}{l}\text { Time from implantation } \\
\text { to transplantation }\end{array}$ & $\boldsymbol{N}$ & $\begin{array}{c}\text { Creatinine } \\
\text { (mg/dL) }\end{array}$ & $\begin{array}{c}\text { Bilirubin } \\
\text { (mg/dL) }\end{array}$ & PRA (\%) \\
\hline 0-2 wk & 59 & $1.61^{*}$ & 2.04 & 2.4 \\
2-4 wk & 58 & 1.18 & $1.55 \dagger$ & 4.7 \\
4-6 wk & 70 & 1.27 & 1.38 & 4.1 \\
6-12 wk & 34 & 1.13 & 1.27 & 5.7 \\
12 wk-6 mo & 71 & 1.15 & 0.98 & 10.0 \\
$>6$ mo & 42 & 1.23 & 0.99 & 11.7
\end{tabular}

$P R A$, Panel-reactive antibody.

${ }^{*} P<.03$ for creatinine less than 2 weeks versus greater than 2 weeks.

$\mathrm{\dagger} P<.0001$ for bilirubin less than 4 weeks versus greater than 4 weeks.

\section{TABLE 7. Creatinine levels (at time of transplantation)}

\begin{tabular}{lc}
\hline Status & Creatinine (mean) \\
\hline Entire cohort & 1.35 \\
1A(a)-VAD & 1.39 \\
1A(a)-other (IABP, ECMO) & 1.49 \\
1A(b)-VAD with complication & 1.34 \\
1A-nonVAD (high inotropes, & 1.45 \\
vent, life $<7 \mathrm{~d}$ ) & \\
1B(a)-VAD $>30 \mathrm{~d}$ & 1.18 \\
1B-nonVAD (low inotropes) & 1.33 \\
2 & 1.30
\end{tabular}

$V A D$, Ventricular assist device; IABP, intra-aortic balloon pump; ECMO, extracorporeal membrane oxygenation.

days without a device-related complication (group $1 \mathrm{~B}[\mathrm{a}]-$ VAD $>30$ days, $P=.009$, Table 7) relative to other groups.

Competing risk: Likelihood of death or removal from waitlist during VAD support. A waiting-list analysis was performed to quantitate the risk of death or removal from the waitlist during VAD support. Among those with a VAD in place at any time $(n=1214)$, the risk of death or removal from the transplantation list was high in the first month after VAD implantation (15.7\% risk of death or removal at 30 days), with a lower risk of death or removal in subsequent months (aggregate risk of death-removal at 6 months was $26.6 \%$, Figure 4). Figure 5 demonstrates time-related outcomes for all patients with a VAD at the time of listing.

\section{Discussion}

This report demonstrates that the interval from VAD implantation to cardiac transplantation is a key determinant of survival in the bridge-to-transplant population. Early device removal and transplantation was associated with a significantly reduced 1-year posttransplantation survival. Multivariate analysis suggests that outcomes after cardiac transplantation are particularly poor in the first 2 to 4 weeks of VAD support and that the window of optimal rehabilitationresuscitation, as measured on the basis of posttransplanta-

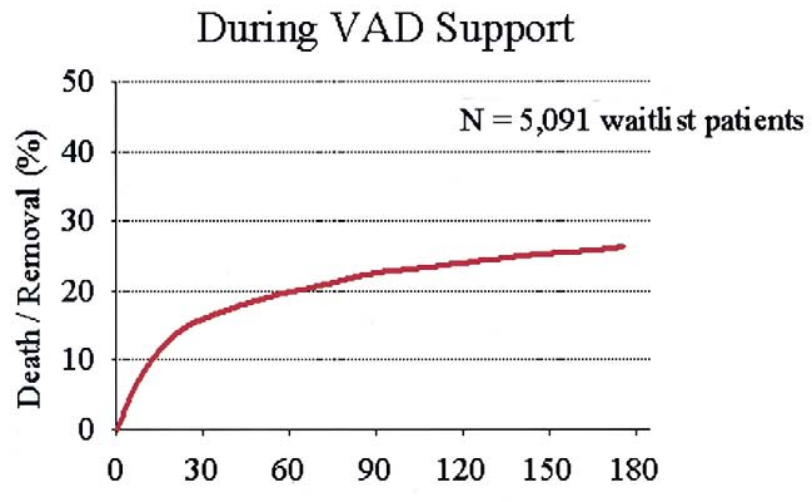

Days of Mechanical Circulatory Support*

Figure 4. Death or removal from the transplantation list during VAD support. *Days of device support, or days since listing if device implanted prior to listing.

tion survival, occurs between 1 and 3 months after VAD implantation. Mechanical support affords the patient with end-stage heart failure the opportunity for reversal of endorgan dysfunction. We found progressive improvement of indices of end-organ function with ongoing mechanical circulatory support.

Although hemodynamic resuscitation occurs almost immediately, restoration of end-organ function and rehabilitation requires weeks of support. We also identified a late phase, during which the benefits of restored end-organ function and overall rehabilitation of physical status were gradually eroded by a progressive increase in device-related complications.

Previous reports have examined the effect of timing of transplantation after VAD implantation, with varying conclusions. Jaski and colleagues ${ }^{4}$ reviewed the Cardiac Transplant Research Database and compared 502 patients supported with VADs with 2514 patients receiving inotropic therapy between 1990 and 1997. There was no difference in posttransplantation survival between the groups. Multivariate analysis did not identify duration of VAD support as a significant determinant of early or late outcome. Ashton and coworkers $^{3}$ reviewed the early clinical experience of 16 centers using the Heartmate LVAD. Among 133 patients successfully bridged to transplantation, there was a 3-fold higher perioperative mortality among those transplanted early ( $<30$ days from VAD implantation), but 1- and 5-year posttransplantation survivals were similar. Kormos and associates $^{7}$ reviewed UNOS data on 167 VAD-supported patients receiving cardiac transplants between 1992 and 1993. Transplantation within 7 days of VAD implantation was an independent predictor of decreased 1-year survival.

Overall, we found 1-year survival after cardiac transplantation to be slightly but significantly lower for the VAD group compared with the non-VAD group. This is in agree- 


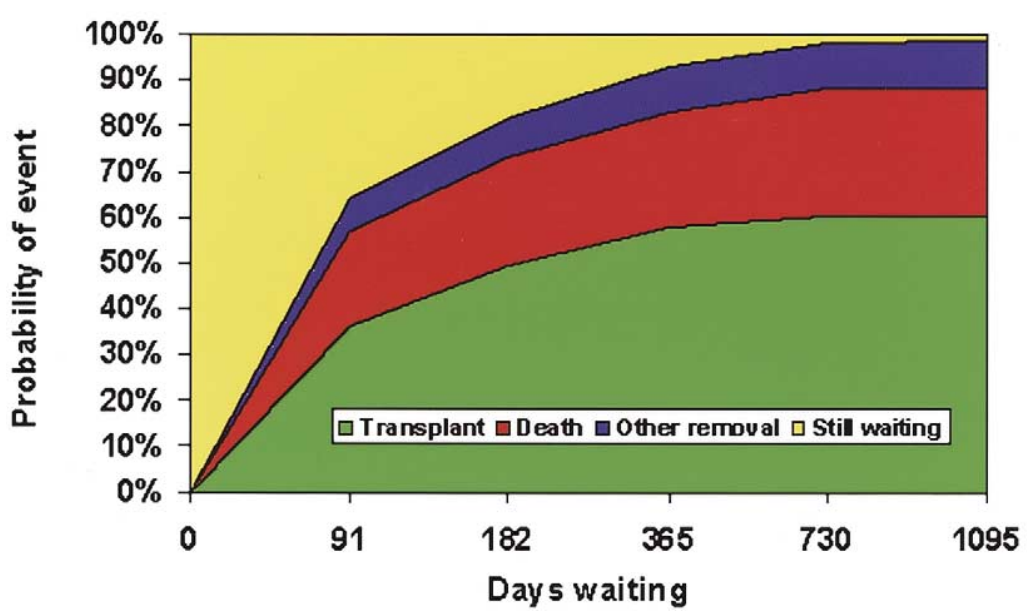

Figure 5. Outcomes on the waiting list for patients with a VAD at listing: adult waiting-list additions from October 25, 1999, to December 31, 2001.

ment with previously reported International Society for Heart and Lung Transplantation registry data, which have consistently demonstrated the presence of a VAD to be a predictor for 1-year mortality (odds ratio, 1.29; $P=.0005$ ) in multivariate analysis. ${ }^{8}$ Other recent single-center reports show equivalent or improved survival after cardiac transplantation for VAD-supported and non-VAD-supported cardiac transplant recipients. ${ }^{1,2}$

In the present series the subgroup of patients supported for more than 30 days free of VAD-related complications had a survival that exceeded that of all other groups of patients. If patients transplanted in the first 2 weeks after VAD insertion were excluded from analysis, 1-year posttransplantation survival was equivalent for the VAD $(84.2 \%)$ and non-VAD (85.7\%) groups. It might be that improved outcomes after VAD bridging reported by experienced centers are the result of a management strategy that explicitly avoids performing cardiac transplantation early after VAD implantation. In the series of Massad and colleagues ${ }^{9}$, median duration of VAD support was 72 days, reflecting a protocol that placed patients on an inactive status for approximately 4 weeks after VAD implantation to allow end-organ recovery. In another recent series that showed superior posttransplantation survival for patients bridged to transplantation with a VAD, only $8.3 \%$ of VAD recipients received a heart transplantation within 30 days of VAD implantation, and the median time to transplantation was 2.9 months. $^{2}$

VAD application in the cardiac transplantation population was evenly distributed on the basis of sex, age, height, weight, and diagnosis. As others have shown, we found that blood group $\mathrm{O}$ patients were more likely to be bridged to transplantation with a VAD, whereas blood group A patients were less commonly bridged. ${ }^{9}$ The median waiting time for a donor heart is 869 days for blood group $\mathrm{O}$ recipients in the United States, which is in contrast to 222 days for blood group A, 152 days for group B, and 68 days for group $\mathrm{AB}$ recipients. ${ }^{10}$ Overrepresentation of blood group $\mathrm{O}$ patients in the VAD population almost certainly reflects a lower threshold for VAD implantation by clinicians who recognize that these patients face a substantial risk of deterioration during protracted waiting times for a donor heart.

As expected, we found that the mean PRA before transplantation was higher in the VAD group than in the nonVAD group. The development of circulating anti-HLA class I and II antibodies (sensitization) occurs more commonly in patients supported with VADs, with some reports showing sensitization in as many as $66 \%$ of bridge-to-transplant patients. ${ }^{1}$ An increased requirement for perioperative blood and blood-product transfusions, device-related infections, and the interaction between the device surface and the patient's immune system are putative mechanisms for the higher rate of sensitization in the VAD-supported population. PRA levels in this study correlated directly with the duration of mechanical circulatory support, increasing from $2.4 \%$ in patients supported for less than 2 weeks to $11.7 \%$ for those undergoing transplantation after more than 6 months of VAD support. It was notable that the mean PRA preceding transplantation was only $7.2 \%$ in this study and that it did not exceed $15 \%$, even among the group supported for more than 6 months.

Device-related complications were common after prolonged device support: nearly one half of patients undergoing transplantation with a device in place were up-classified to status $1 \mathrm{~A}$ as a result of a device-related complication occurring more than 30 days after VAD implantation. Only $16 \%$ of the bridged population enjoyed prolonged $(>30$ days) device support without complications. This experience is similar to that of other reports of patients undergoing 
long-term VAD support. Late device-related complications were common in the REMATCH study, with an incidence of sepsis and suspected LVAD malfunction of 0.60 and 0.75 events per patient-year, respectively. ${ }^{11}$

We found improvement in measures of renal and hepatic function with ongoing mechanical circulatory support. There was a similar degree of renal dysfunction among all patient groups undergoing cardiac transplantation (mean creatinine level, $1.35 \mathrm{mg} / \mathrm{dL}$ ), except the group of patients bridged to transplantation with a VAD for more than 30 days free of device-related complications (1B[a]-VAD $>30$ days). The mean creatinine level in this group was significantly less $(1.18 \mathrm{mg} / \mathrm{dL}, P=.009)$ than all other groups. These findings support the notion that weeks of hemodynamic support are required to achieve normalization of end-organ function and are concordant with prior reports that have demonstrated improvement of both hepatic and renal function during long-term VAD support in cohorts of patients bridged to transplantation., ${ }^{9,12}$

There is a continued reliance on VADs to bridge patients with end-stage heart failure to transplantation. During the time period examined in this study, $17 \%$ of recipients in the United States were bridged to cardiac transplantation with a VAD. Previous studies have demonstrated a growing use of VADs in the cardiac transplantation population. Among participants in the Cardiac Transplant Research Database, application of VADs increased from $2 \%$ to $16 \%$ between 1990 and 1997. ${ }^{4}$ Other large single-center experiences have reported VAD support in up to $43 \%$ of recipients. ${ }^{1,13}$ Our analysis provides evidence-based guidance regarding timing of transplantation that might result in improved long-term outcomes for a significant portion of the cardiac transplant population.

There are several limitations of this study. This study was retrospective and nonrandomized. The timing of transplantation in each case was determined by clinicians seeking to optimize the outcome of individual patients. Although we have shown a strong relationship between the timing of transplantation and outcome, it is possible that there were confounding factors that our analysis did not take into consideration. The small number of patients supported with an Abiomed device were more likely to undergo transplantation within 30 days of implantation than those supported with other devices, as would be expected given the Abiomed's design for short-term support. Selection of sicker patients for support with this device might have contributed to poorer outcomes among patients undergoing transplantation early after device implantation. Because of changes in UNOS data-gathering procedures, data regarding the time interval from VAD implantation to cardiac transplantation were not available for $28 \%$ (132/466) of the population studied. We compared both recipient and donor factors for the patients with and without time interval data and found few differences, suggesting that our analyses are representative of the entire population (see the Appendix).

\section{Conclusion}

Our study clearly demonstrates that cardiac transplantation should be deferred for a minimum of 2 to 4 weeks after VAD implantation, if clinical circumstances permit, to allow rehabilitation and recovery of end-organ function. Ideally, transplantation of the VAD-supported patient should occur when the recipient is extubated, ambulating, eating, and outside of the intensive care unit and has normalization of renal and hepatic function. Our data support the hypothesis that avoidance of transplantation early after VAD insertion yields improved survival after transplantation. Under the 1999 UNOS rules, a substantial number of patients in the United States underwent transplantation within 1 month of device insertion. Recent UNOS rule changes have given the clinician discretion to upgrade a device-dependent patient to status $1 \mathrm{~A}$ for a 30-day period at any time after $\mathrm{VAD}$ insertion. This change will further encourage timing of transplantation to coincide with maximal patient rehabilitation.

\section{References}

1. John R, Lietz K, Schuster M, Naka Y, Rao V, Mancini DM, et al. Immunologic sensitization in recipients of left ventricular assist devices. J Thorac Cardiovasc Surg. 2003;125:578-91.

2. Aaronson KD, Eppinger MJ, Dyke DB, Wright S, Pagani FD. Left ventricular assist device therapy improves utilization of donor hearts. $J$ Am Coll Cardiol. 2002;39:1247-54.

3. Ashton RC Jr, Goldstein DJ, Rose EA, Weinberg AD, Levin HR, Oz MC. Duration of left ventricular assist device support affects transplant survival. J Heart Lung Transplant. 1996;15:1151-7.

4. Jaski BE, Kim JC, Naftel DC, Jarcho J, Costanzo MR, Eisen HJ, et al. Cardiac transplant outcome of patients supported on left ventricular assist device vs. intravenous inotropic therapy. J Heart Lung Transplant. 2001;20:449-56.

5. Renlund DG, Taylor DO, Kfoury AG, Shaddy RS. New UNOS rules: historical background and implications for transplantation management. United Network for Organ Sharing. J Heart Lung Transplant. 1999;18:1065-70.

6. Arriagada R, Rutqvist LE, Kramar A, Johansson H. Competing risks determining event-free survival in early breast cancer. $\mathrm{Br} J$ Cancer. 1992;66:951-7.

7. Kormos RL, Breen TJ, Frazier OH, Hosenpud JD. Sub-study analysis of ISHLT/UNOS Registry, Richmond, VA: use of ventricular assist devices before heart transplant effects of type of support on transplant survival. J Heart Lung Transplant. 1995;14(1:2):S67.

8. Hosenpud JD, Bennett LE, Keck BM, Boucek MM, Novick RJ. The Registry of the International Society for Heart and Lung Transplantation: eighteenth official report-2001. J Heart Lung Transplant. 2001; 20:805-15.

9. Massad MG, McCarthy PM, Smedira NG, Cook DJ, Ratliff NB, Goormastic M, et al. Does successful bridging with the implantable left ventricular assist device affect cardiac transplantation outcome? J Thorac Cardiovasc Surg. 1996;112:1275-83.

10. UNOS/OPTN annual report. Available at: www.optn.org/data/ annualreport. Accessed 2003.

11. Rose EA, Gelijns AC, Moskowitz AJ, Heitjan DF, Stevenson LW, Dembitsky W, et al. Long-term mechanical left ventricular assistance for end-stage heart failure. N Engl J Med. 2001;345:1435-43.

12. Frazier OH, Macris MP, Myers TJ, Duncan JM, Radovancevic B, 
Parnis SM, et al. Improved survival after extended bridge to cardiac transplantation. Ann Thorac Surg. 1994;57:1416-22.

13. McCarthy PM, Savage RM, Fraser CD, Vargo R, James KB, Goormastic $\mathrm{M}$, et al. Hemodynamic and physiologic changes during support with an implantable left ventricular assist device. J Thorac Cardiovasc Surg. 1995;109:409-18.

\section{Discussion}

Dr Francis D. Pagani (Ann Arbor, Mich). Dr James Gammie and colleagues have presented an excellent manuscript on the influence of duration of VAD support on survival after heart transplantation. These authors observed that the shorter durations of VAD support adversely affected heart transplantation survival as a result of incomplete recovery of recipient end-organ function. Additionally, longer periods of VAD support were also associated with reduced posttransplantation survival, presumably as a consequence of complications of extended VAD support, such as allosensitization. I have several comments and questions for Dr Gammie and his colleagues.

Of the 466 patients on VAD support at the time of transplantation identified in the SRTR registry, $126(27 \%)$ of the patients had no date of LVAD implantation recorded in the SRTR registry database. In the analysis of the influence of duration of VAD support on heart transplantation survival depicted in slide 13 of the authors' presentation, only 334 patients, and not 466 patients, appeared to have been included for analysis of the effects of the duration of VAD support on heart transplantation survival. My first question is, could the exclusion of $27 \%$ of the patients with VAD support have added an important bias to the results of the data analysis?

Intuitively, all surgeons understand that increasing degrees of organ dysfunction increase operative risk, and thus it is important to understand the reasoning behind the decision to proceed with heart transplantation early after VAD insertion despite the presence of significant recipient organ dysfunction.

Do the authors know what proportion of the 169 patients who underwent heart transplantation within the first month after VAD insertion were supported by a VAD not intended to bridge long term, such as an Abiomed or centrifugal device, or a hybrid combination of devices, such as an Abiomed RVAD and a HeartMate LVAD, for those patients who experience right-sided circulatory failure? These scenarios would obviously increase the likelihood of a transplant surgeon accepting a donor organ for a recipient patient despite the presence of significant organ dysfunction.

Did the authors perform a similar analysis of the effects of the duration of VAD support by using only those patients who were supported with an intracorporeal LVAD device, such as the HeartMate or Novacor device?

Importantly, did the authors include VAD type as a factor in their multivariate proportional hazards model in analyzing for posttransplantation survival?

Dr Gammie and colleagues were careful to analyze donor characteristics between patients on VAD support and those transplanted without VAD support to account for differences in survival. In addition, the influence of heart donor characteristics on heart transplant recipient survival within the groups of patients with different time intervals of VAD support was also investigated.
However, because of the limitations of the SRTR registry, many important donor characteristics were absent from the analysis.

Is it likely that the group of patients who underwent heart transplantation early after initiation of VAD support were more likely to receive a marginal heart donor? For example, was a patient undergoing Abiomed support or support under hybrid circumstances more likely to receive the first available organ, even if that organ was of marginal status?

I would like to congratulate Dr Gammie and his colleagues for a well-conceived and excellent presentation on a timely topic. These data improve our understanding of the optimal time frame to allocate status $1 \mathrm{~A}$ time to patients with VAD support under the current UNOS policy.

Dr Gammie. Thank you very much for your kind comments, Dr Pagani. Many of them relate to the limitation of our data set, and I do acknowledge, and we acknowledge in the abstract, as well as in the manuscript, that there was some degree of missing data. The strength of this data set is that it is large and encompasses all institutions in the United States. The limitations are exactly as you have mentioned.

During the time period of the study, the method of data collection changed. Early on in the study, data were collected on paper forms for compliance reasons, and then as the study progressed, data were collected electronically through the UUNET system. And therefore we believe that the missing data, specifically the time interval from VAD insertion to transplantation, was a time-related phenomenon and that there was no specific bias. And in our manuscript we actually went back and compared those patients who had missing data with the remainder who had time data, and we found no significant differences in those 2 populations. Therefore certainly a sampling error could exist, but on the basis of our analyses, we think that our analyses are based on a fairly representative group of patients.

You asked which proportion of those patients undergoing transplantation early after VAD insertion were on an Abiomed, a device that, compared with the other devices in our study, is designed for probably a shorter-term support. That is a good question, and in fact, we are going back and looking at that. It looks as though there were about 40 patients supported on Abiomed during the first month, and we did not include that in our multivariate analysis, but that is an excellent suggestion, and we will go ahead and look at that.

In terms of the donor data, admittedly, we did not have ejection fraction, but all other characteristics, including ischemic time and age and other factors, looked as though they were similar in the different groups.

Dr O. Howard Frazier (Houston, Tex). I appreciate this interesting collection of data. The first LVAD to be approved by the US Food and Drug Administration (FDA), which is a third-party analysis of the data, used a control group of 36 patients who met all the indications for implantation, but the device was simply not available. Only 12 of those patients were transplanted-the rest died-with the longest surviving 2 weeks, and 9 of those patients survived. The 12 patients undergoing transplantation had an average waiting time of 5 days.

As for the patients who received the LVAD, this group, from 1988 through 1992, had a $70 \%$ survival to transplantation, and the 
duration of implantation was 132 days in that group, with a $90 \%$ 1-year survival. These are the data that we presented to the panel that was connected with the FDA. Therefore they were very good data. There was less rejection in these patients as well.

A similar panel was contrived for the electric LVAD group, which was approved in 1996. There were 240 of those patients, and they had an $84 \%$ one-year survival after transplantation, with a mortality similar to that of the control groups.

First, I think it is good to classify these devices. One of the reasons I got involved in UNOS again was because of this accelerated status, and in fact, I know the Abiomed results and I know the 30-day results, and those are what prompted us to change that classification because that is the period when the device should not be implanted. The patients need to recover and that is what we learned over a decade ago. The poor results also might be correlated to the type of device, as was previously stated.

Do you think that some of these poorer results that we have seen are a reflection of the larger number of centers? In one of the earlier studies, the centers that were involved in this were limited and very experienced, and there is a high learning curve to the use of these technologies.
This was an excellent presentation, and it is of some interest, but I think it is very important not to compare the LVAD groups with the routine transplantations if the only indication for an LVAD is imminent mortality.

Dr Gammie. Thank you, Dr Frazier. I am honored by your comments, and indeed, a decade ago you showed us all the benefits of prolonged device support in terms of restoration and of endorgan function and exceptionally good outcomes after heart transplantation in a group of patients who, as you pointed out, were indeed sicker. I certainly think there probably is a center volumeoutcome relationship, although we did not specifically look at that.

One thing I would like to point out is that although singlecenter results have shown very good outcomes, those centers certainly stay away from transplanting patients in the first 30 days, and if you look carefully at the literature, very few patients undergo transplantation in that first 30 days, whereas in our experience here, up to a third of patients indeed underwent transplantation in the first 30 days.

Therefore I think the lesson is you probably should stay away from that time frame, if possible recognizing the complexity of each individual patient. 
APPENDIX TABLE 1. Comparison of donor and transplant characteristics between transplantations in VAD-supported patients with and without time from implantation to transplantation

\begin{tabular}{|c|c|c|c|}
\hline & $\begin{array}{l}\text { Missing time } \\
\quad(n=132)\end{array}$ & $\begin{array}{c}\text { Time known } \\
(\mathrm{n}=334)\end{array}$ & $P$ valuet \\
\hline \multicolumn{4}{|l|}{ Characteristic $^{*}$} \\
\hline Age (y) & 50.30 & 50.61 & .8 \\
\hline Most recent PRA & 9.22 & 6.34 & .1 \\
\hline Most recent creatinine & 1.48 & 1.27 & .04 \\
\hline Donor age (y) & 32.67 & 32.40 & .8 \\
\hline Ischemia time (h) & 3.25 & 3.05 & .2 \\
\hline Donor total bilirubin (mg/dL) & 0.94 & 1.00 & .4 \\
\hline Donor serum creatinine $(\mathrm{mg} / \mathrm{dL})$ & 1.02 & 1.05 & .4 \\
\hline \multicolumn{4}{|l|}{ Donor characteristic $\ddagger$} \\
\hline \multicolumn{4}{|l|}{ ABO } \\
\hline$A$ & $36.4 \%$ & $34.7 \%$ & .7 \\
\hline 0 & $48.5 \%$ & $48.2 \%$ & .9 \\
\hline Female & $19.7 \%$ & $23.7 \%$ & .4 \\
\hline \multicolumn{4}{|l|}{ VAD type } \\
\hline Abiomed & $3.2 \%$ & $9.8 \%$ & \\
\hline Novacor & $18.3 \%$ & $9.8 \%$ & \\
\hline Heartmate & $64.5 \%$ & $61.2 \%$ & .04 \\
\hline Thoratec & $10.8 \%$ & $16.7 \%$ & \\
\hline Other & $3.2 \%$ & $2.5 \%$ & \\
\hline \multicolumn{4}{|l|}{ Cause of death } \\
\hline Anoxia & $2.3 \%$ & $8.4 \%$ & \\
\hline Cerebrovascular & $36.6 \%$ & $29.6 \%$ & \\
\hline Head trauma & $58.8 \%$ & $60.5 \%$ & .06 \\
\hline CNS tumor & $1.5 \%$ & $1.2 \%$ & \\
\hline Other & $0.8 \%$ & $0.3 \%$ & \\
\hline Clinical infection at time of recovery & $28.0 \%$ & $24.6 \%$ & .5 \\
\hline Cigarette use $>20$ pack-years & $36.7 \%$ & $38.4 \%$ & .7 \\
\hline History of cocaine use§ & $14.3 \%$ & $15.0 \%$ & .8 \\
\hline Pretreatment steroids & $54.3 \%$ & $56.2 \%$ & .7 \\
\hline History of diabetes & $1.5 \%$ & $2.7 \%$ & .7 \\
\hline History of hypertension & $12.3 \%$ & $13.0 \%$ & .8 \\
\hline
\end{tabular}

NOTE: Percentages were computed on the basis of known values only.

$V A D$, Ventricular assist device; PRA, panel-reactive antibody; $C N S$, central nervous system.

*Mean per group.

†Percentage within group.

$\ddagger P$ values were computed by using the Wilcoxon test for continuous factors and the $\chi^{2}$ test for categoric factors, except for VAD type and cause of death. Because of the relatively small number of transplantations in some cells, the Fisher exact test was used for these 2 factors.

§Cocaine use collected on donors only since October 25, 1999. 